\title{
SHOCK WAVE PROPAGATION IN A DOUBLE ROOM
}

\author{
GAETAN RUSCADE ${ }^{1}$, ISABELLE SOCHET ${ }^{1}$, LUC HAKENHOLZ ${ }^{2}$ \& KARIM DJAFER ${ }^{2}$ \\ ${ }^{1}$ Institut National des Sciences Appliquées Centre Val de Loire, Université d'Orléans, France \\ ${ }^{2}$ DGA Techniques Navales, France
}

\begin{abstract}
The present research focuses on the propagation of a shock wave in a double room divided by a wall in which there is an opening. It is experimental work conducted on a small scale. The shock wave is generated by the detonation of a gaseous charge located in the emitter room. The model is equipped with pressure sensors in both rooms. Here, the effect of the geometry and dimensions of the opening in the connecting wall on the reflection of shock waves is analysed in the receptor room. The shock wave parameters in terms of overpressure and arrival time were determined for the three reflected peaks.

Keywords: blast wave, confined explosion, multi-room, shock reflection.
\end{abstract}

\section{INTRODUCTION}

Accidental or intentional explosions inside public, industrial or military infrastructures have become an important safety issue. Inside a confined environment, the interactions between the blast and the geometry, such as reflection and diffraction, make the interpretation of blast wave phenomena highly complex. That is why the understanding of shock wave parameters is essential for the design of military infrastructures such as warships or submarines.

Numerous studies are related to shock wave propagation in confined environments: some of them concern the influence of afterburning (Togashi et al. [1], for example), jetting in corridors (van Erkel [2], for example), or venting explosions (Salvado et al. [3], for example).

To understand the loading inside a building or a model, it is important to have knowledge about indoor interactions. The most common effects are the increase or the mitigation of the overpressure and impulse due to reflection, recombination, diffraction or ceiling effects. First of all, shock wave reflection, described experimentally by Ben-Dor [4], can increase shock wave parameters in some areas of the model. Some studies have been conducted that focus on multi-reflection inside a confined chamber. Sugiyama et al. [5] studied blast waves inside a chamber with an exit, and defined a parameter to determine the overpressure at the exit. Mirova et al. [6] performed an experiment on multi-reflection inside a shock-tube, as did Teland et al. [7], comparing experimental results with simulation since inconsistencies between inside blast simulation and experiments were observed.

Lastly, multi room investigations are conducted for safety purposes, such as transport (Gebbeken et al. [8] for subways), or energy installations (Miura et al. [9] in a Nuclear Fuel Cycle Facility). In the present paper, experiments will be used for a military purpose, to estimate the effect of a load on double rooms inside a warship or a submarine in the event of explosion on board.

A first study was carried out by Gault [10] on a single chamber, and a predictive code based on experimental laws was developed. The small-scale model used for experiments is made of two different chambers, separated by different sized openings. The goal is to combine the effect of the gaseous charge position, the size and the direction of the opening and the prediction of reflected peaks. 


\section{DESCRIPTION OF THE EXPERIMENTAL SETUP}

The main goal of this study was to determine laws that can describe the main characteristics of shock waves, such as overpressure, impulse and arrival time, in a multi-room environment. To do so, several tests were performed in a small-scale model.

\subsection{Model}

The model is composed of two polycarbonate boxes separated by a plate with different openings (Fig. 1). The emitting chamber is where the charge is located, and the receiver chamber is where most of the sensors are placed. The dimensions of the two cubes are the following: $0.53 \mathrm{~m} \times 0.50 \mathrm{~m} \times 0.50 \mathrm{~m}$ for the first chamber (with the charge) and $0.50 \mathrm{~m} \times$ $0.50 \mathrm{~m} \times 0.50 \mathrm{~m}$ for the receiver chamber. The thickness of all the polycarbonate plates and all the walls is $0.03 \mathrm{~m}$. The model is bolted onto an aluminium bench $(1.30 \mathrm{~m} \mathrm{x} 0.76 \mathrm{~m}$ x $0.05 \mathrm{~m})$.

Twelve different plates were made to study the influence of the surface and the direction of the opening, six with a horizontal blowhole and six with a vertical one. Fig. 2 shows an example of each one. The different openings are named: $\mathrm{PH}(\mathrm{V})-300$, where $\mathrm{H}$ means horizontal and $\mathrm{V}$ vertical for the direction and the number represents the surface of the opening (in $\mathrm{cm}^{2}$ ). All the plates have a constant coaming of $0.03 \mathrm{~m}$. The characteristics of the openings are summarized in Table 1.

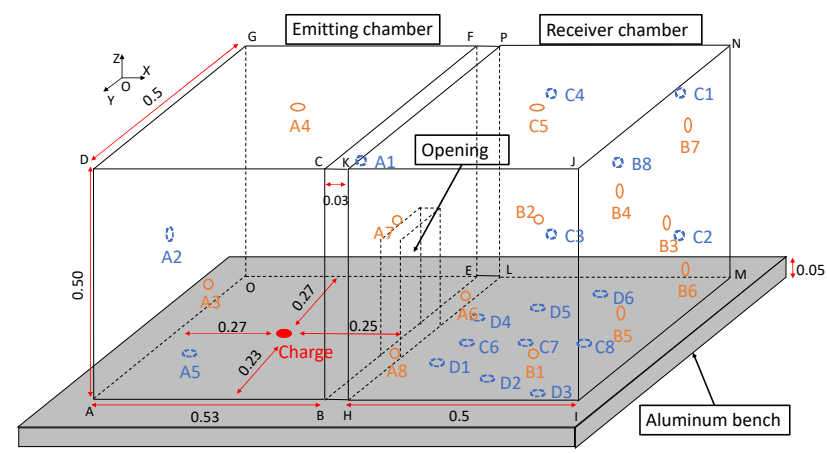

Figure 1: Representation of the small-scale model with sensors (units: meters).

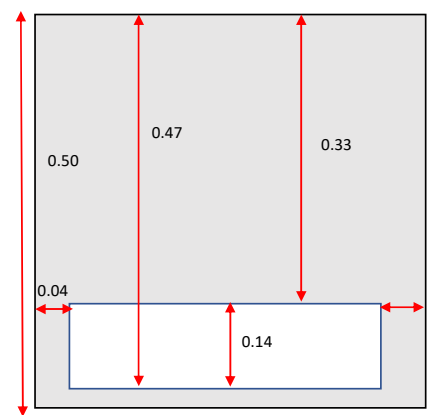

$\mathrm{PH}-588$

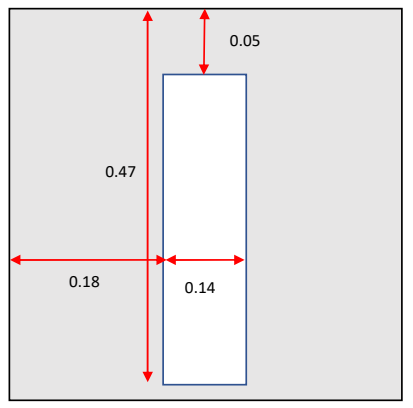

PV-588

Figure 2: Outline of one vertical opening and one horizontal one. 
Table 1: Characteristics of horizontal and vertical openings.

\begin{tabular}{|l|r|r|r|r|r|r|}
\hline Opening name & PH-300 & PH-507 & PH-588 & PH-840 & PH-1050 & PH-1344 \\
\hline Opening name & PV-300 & PV-507 & PV-588 & PV-840 & PV-1050 & PV-1344 \\
\hline S $_{\text {Opening }}\left(\mathrm{m}^{2}\right)$ & 0.0300 & 0.0507 & 0.0588 & 0.0840 & 0.1050 & 0.1344 \\
\hline
\end{tabular}

\subsection{Charge and sensors}

Fig. 3 shows the matrix of positions for the charge. Two positions for the charge were studied: in blue is the first position used in this study, named P1, and in red the second one P2. The sensor A5, that is used for the trigger and as a reference to validate the pressure levels, is shown in green.

Experiments took place at ambient pressure and temperature. The charge used is a gaseous stoichiometric mixture of propane oxygen, and the radius of the gas confinement bubble is $0.05 \mathrm{~m}$. The corresponding gaseous mass is a hemispherical charge of $0.369 \times 10^{-3} \mathrm{~kg}$. The nominal energy of ignition is $8 \mathrm{kV}$.

To record the pressure signal in the model, 30 sensors were used, 20 Kistler $603 \mathrm{C}$ and 10 Kistler 603B. 603B are useful to record the ground pressure signal from the second box, they are less sensitive to vibration than $603 \mathrm{C}$. The fact that two kind of sensors were used does not impact result repeatability.

\section{LAWS}

The study of incident and reflected shock waves inside a two-room model is not that simple. We therefore restricted our research on the shock wave parameters to the four overpressure peaks, as well as the maximum of overpressure. It is first necessary to have criteria to select the first four overpressure peaks.

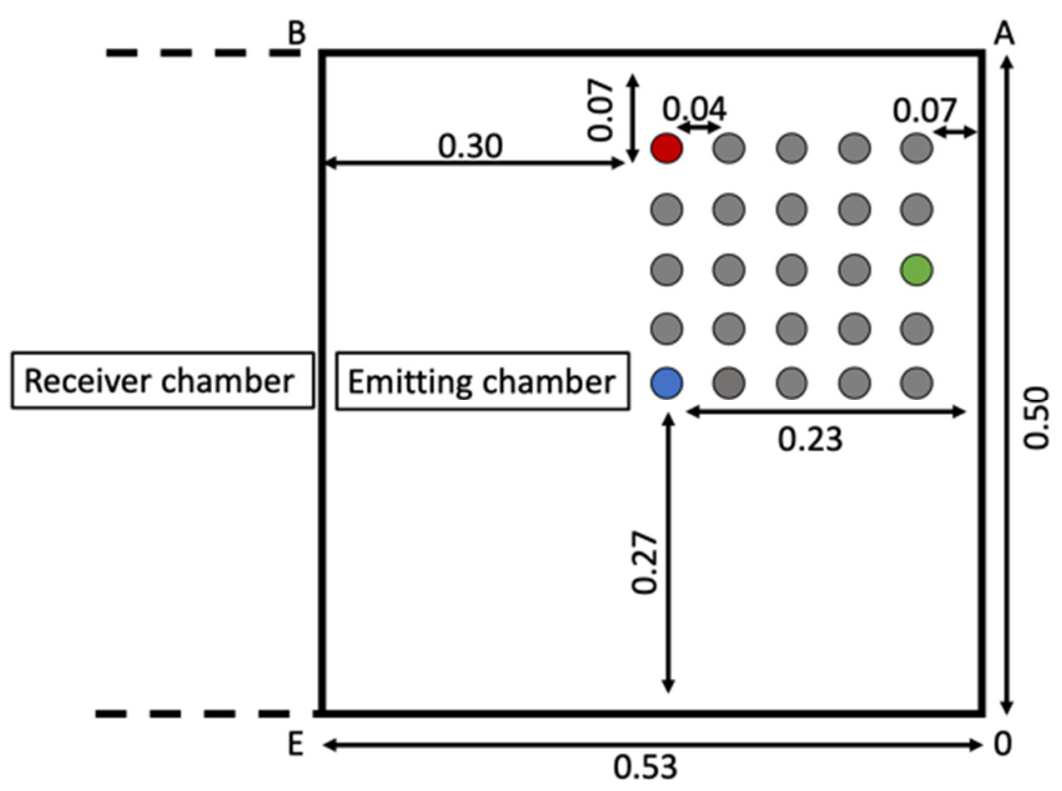

Figure 3: Mapping of the sensors and the charge matrix position on the floor. 
The main goal of this study was to develop experimental laws from all the data gathered during tests inside the model. A simple predictive and fast-running tool will then be computed based on experimental laws, to determine the path and the main characteristics of shock waves inside a two-room model. These laws focus on the arrival time of the first four overpressure peaks and the maximum overpressure.

\subsection{Reflection peak criteria}

To achieve a good understanding of the phenomena inside the model, it was important to analyse all the pressure profiles for both charge positions and to compare them. To choose the reflected peaks and to automate their selection a Matlab code was developed. The following criteria were found to be the most accurate at finding experimental laws and were therefore selected:

- Number of peaks: four reflections are considered.

- Minimum overpressure: $\Delta \mathrm{Pmin}=0.05$ bar.

- Amplitude of the overpressure peak: 0.05 bar.

- Time difference between two peaks: $0.1 \mu \mathrm{s}$.

\subsection{Parameters}

Three parameters were used to find the different laws:

- The first one is the scaled distance $\mathrm{Z}\left(\mathrm{m} \cdot \mathrm{kg}^{-1 / 3}\right)$, defined by the following equation:

$$
\mathrm{Z}=\mathrm{R} \cdot \mathrm{M}^{-1 / 3} \text {, }
$$

where $\mathrm{R}$ is the distance between the charge and the sensor, and $\mathrm{M}$ the mass of the gaseous charge.

- The second one represents the geometry of the opening, $\mathrm{H} / \mathrm{L}$, where $\mathrm{H}$ is the height of the opening and $\mathrm{L}$ the width.

- The third is the angle of incidence between the normal to the wall and the direction charge-sensor. It is named $\theta$, as shown in Fig. 4.

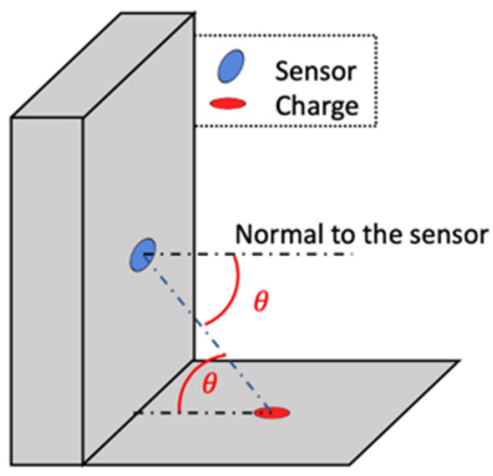

Figure 4: Definition of angle parameters $-\theta$. 


\subsection{Arrival time}

In the case of arrival time, the four of reflected peaks are separated, and each one has its own law. Figs 5, 6, 7 and 8 show the different arrival time correlations.

The first arrival time is plotted as a function of the scaled distance. For the others, the parameter $\mathrm{H} / \mathrm{L}$ is used: the arrival time is multiplied by $\mathrm{H} / \mathrm{L}$ and plotted versus the scaled distance also multiplied by H/L. For each arrival time a lower and a higher correlation are defined to ensure a $95 \%$ confidence interval.

For the first arrival time, the following eqns were found:

$$
\begin{gathered}
t a_{1}=0.2116 Z-0.5549 \\
t a_{1 \_ \text {low }}=0.2090 Z-0.5804 \\
t a_{1 \text { _high }}=0.2023 Z-0.4909
\end{gathered}
$$

with $4.71 \mathrm{~m} \cdot \mathrm{kg}^{-1 / 3} \leq \mathrm{Z} \leq 14.22 \mathrm{~m} \cdot \mathrm{kg}^{-1 / 3}$ and $t a_{1}$ in ms.

Eqn (2) is for the general law, with a correlation coefficient of 0.974. Eqn (3) represents the lower correlation and eqn (4) the higher correlation.

As shown in Fig. 5, some areas of the chart exhibit a significant error, with a $10 \%$ deviation from the law. An identification study was conducted to find out which sensors were responsible for these errors. The points inside the red circle represent sensor A7, which is the one closest to the vertical opening. The green circle corresponds to sensors located on the top half of the back face (sensors B4-B7) for small openings (PH-300/PV-300). The blue one refers to sensors C1-B2 located in the top half of the side walls.

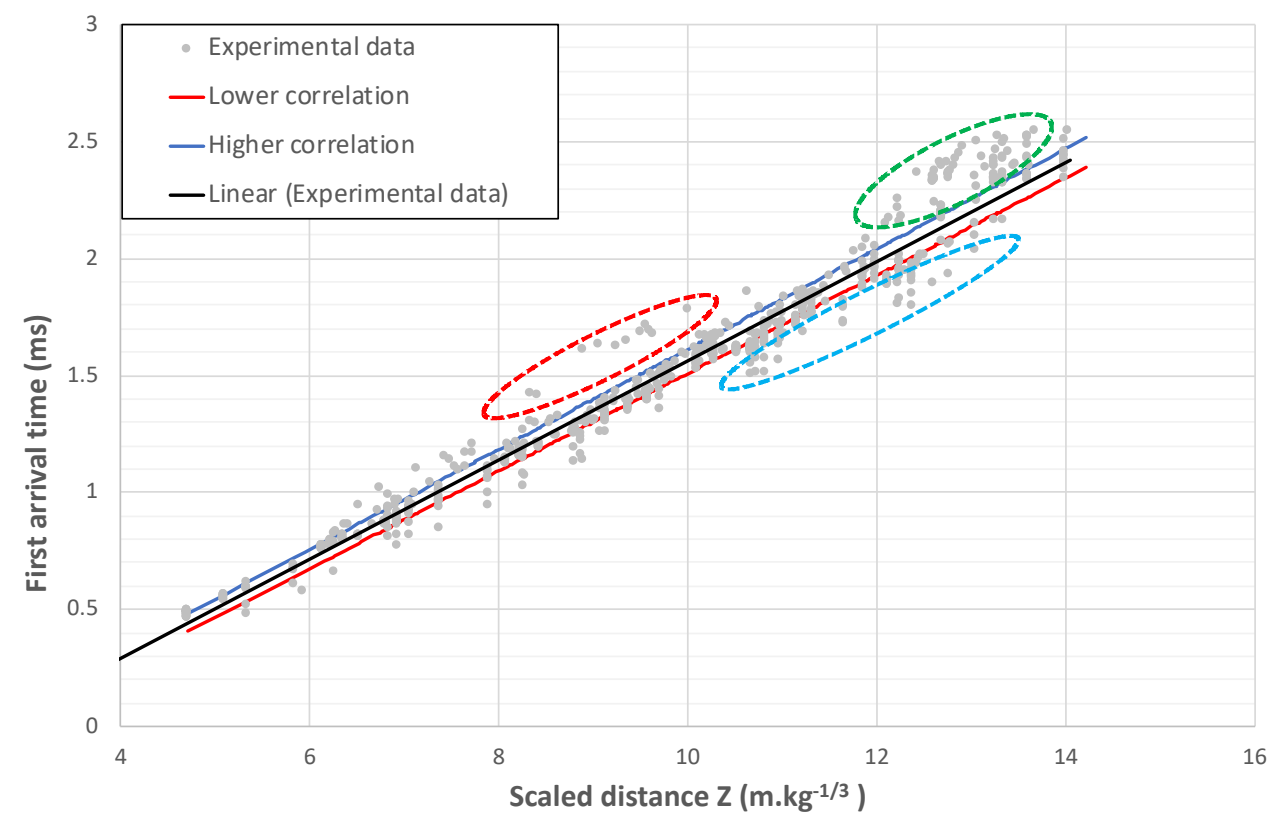

Figure 5: Correlation on the arrival time for the first reflected peak. 


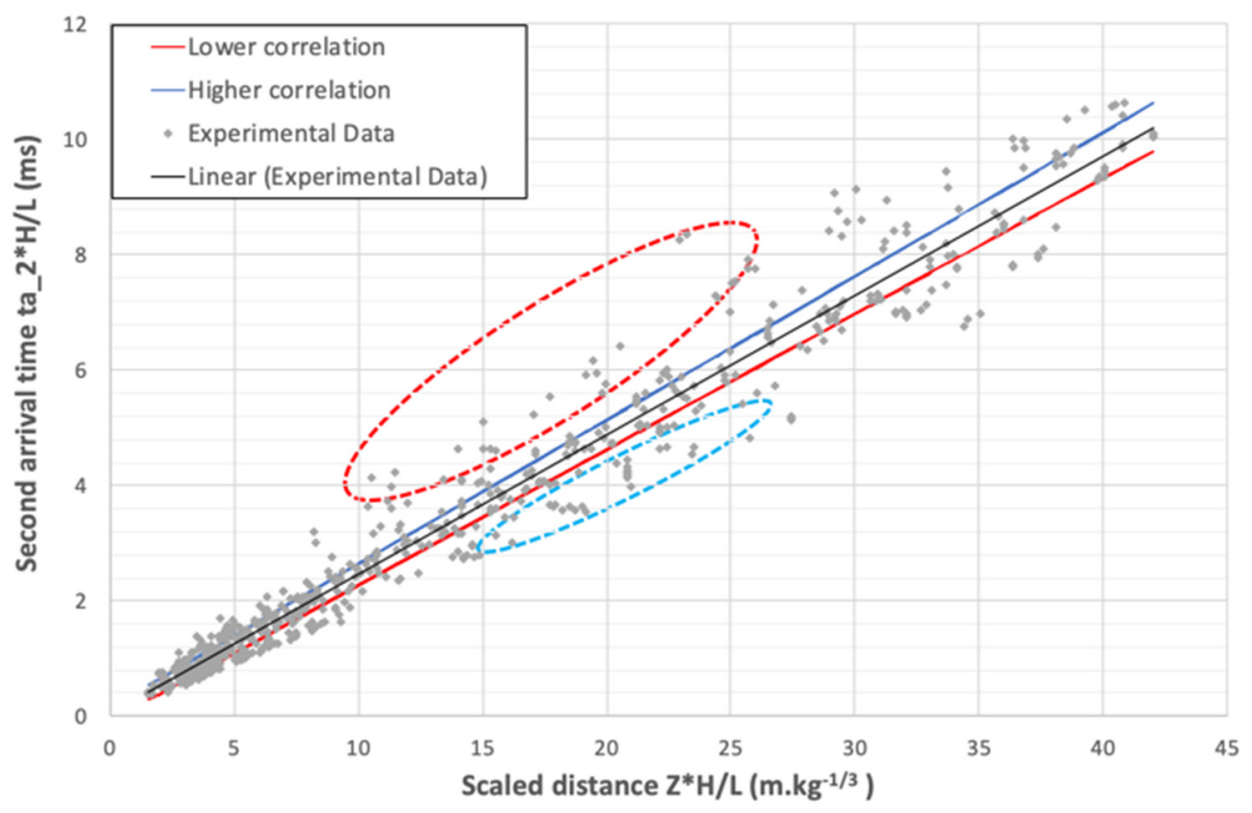

Figure 6: Correlation on the arrival time for the second reflected peak.

Regarding the second arrival time, the following equations were found:

$$
\begin{gathered}
t a_{2} * \frac{H}{L}=0.2428 Z * \frac{H}{L}-0.0368 \\
t a_{2 \_l o w} * \frac{H}{L}=0.2357 Z * \frac{H}{L}-0.0906 \\
t a_{2 \_ \text {high }} * \frac{H}{L}=0.2493 Z * \frac{H}{L}+0.1481
\end{gathered}
$$

with $4.71 \mathrm{~m} \cdot \mathrm{kg}^{-1 / 3} \leq \mathrm{Z} \leq 14.22 \mathrm{~m} \cdot \mathrm{kg}^{-1 / 3}$ and $t a_{2}$ in ms.

The same identification study was conducted. In Fig. 6, the red circle represents sensors A7-A8-A6, positioned close to the vertical opening. The blue circle corresponds to sensors located on the bottom half of the model (sensors B1-B6-B5-C1) and on the ground for vertical openings.

The dispersion increases for the third and the fourth arrival times. That is why in addition to the lower and higher correlations that defined a $95 \%$ confidence interval, two laws were defined with a $99 \%$ confidence interval.

All the laws can be written as ta $* \frac{\mathrm{H}}{\mathrm{L}}=\mathrm{A} * \mathrm{Z} * \frac{\mathrm{H}}{\mathrm{L}}+\mathrm{B}$ with coefficients $\mathrm{A}$ and $\mathrm{B}$ depending on the law. Table 2 summarizes these coefficients. Fig. 7 and Fig. 8 represent the evolution of the third and fourth arrival times.

For the third peak, the maximum error (circle in Fig. 7) is equal to a $35 \%$ deviation from the law. It corresponds to sensor C3 with the opening PV-300 in charge position 2. For the fourth peak, the error is a $50 \%$ deviation from the law, for sensor A8, with the opening PV-588 in charge position 2. 
Table 2: Characteristics of correlations on the arrival time for the third and fourth peaks.

\begin{tabular}{|c|c|c|c|c|}
\hline & Kind of correlation & A & B & $\begin{array}{l}\text { Coefficient of } \\
\text { determination }\end{array}$ \\
\hline \multirow{5}{*}{ 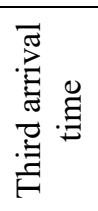 } & Medium & 0.3086 & 0.186 & \multirow{5}{*}{$R^{2}=0.875$} \\
\hline & Lower correlation & 0.2983 & -0.0022 & \\
\hline & Higher correlation & 0.3190 & 0.3622 & \\
\hline & Lower Law & 0.2523 & -0.0082 & \\
\hline & Higher Law & 0.3581 & 0.4012 & \\
\hline \multirow{5}{*}{ 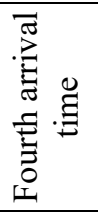 } & Medium & 0.3629 & 0.3719 & \multirow{5}{*}{$\mathbf{R}^{2}=\mathbf{0 . 8 2 3}$} \\
\hline & Lower correlation & 0.3499 & 0.1451 & \\
\hline & Higher correlation & 0.3759 & 0.5989 & \\
\hline & Lower Law & 0.2889 & -0.3324 & \\
\hline & Higher Law & 0.4120 & 0.7001 & \\
\hline
\end{tabular}

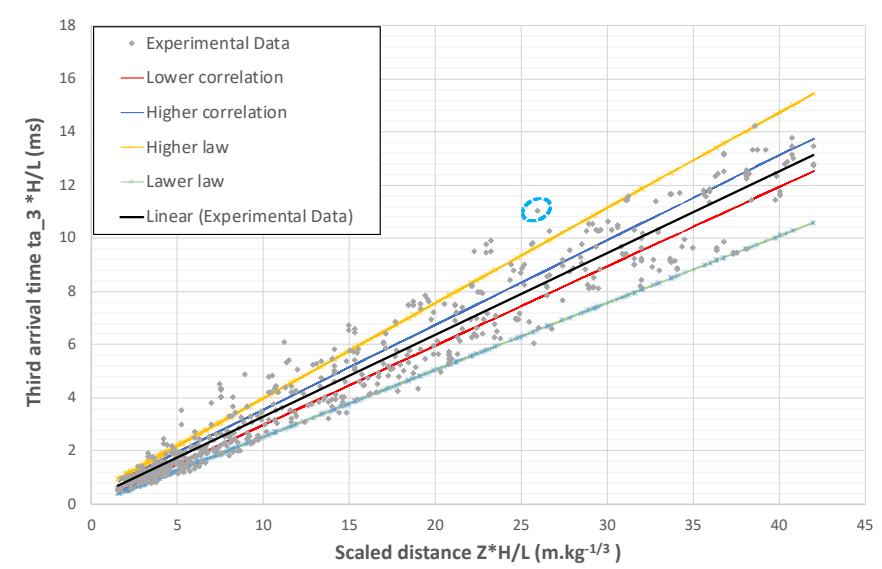

Figure 7: Correlation on the arrival time for the third reflected peak.

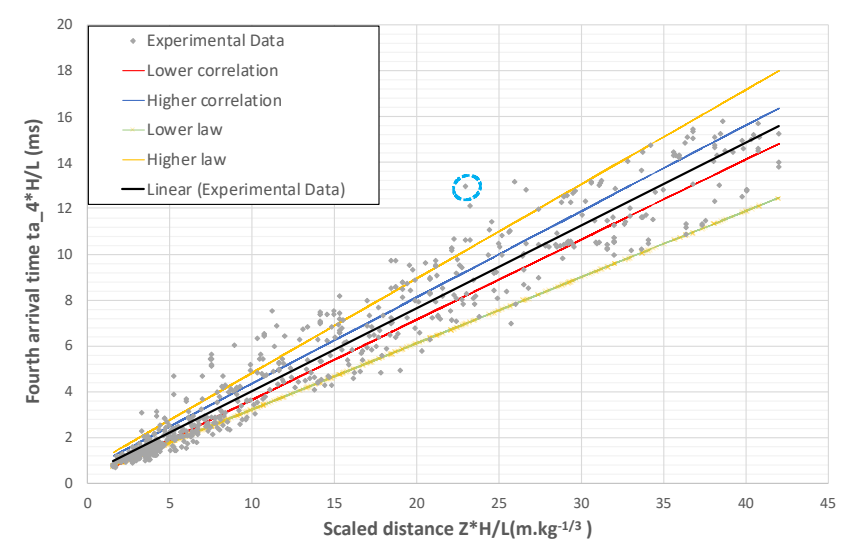

Figure 8: Correlation on the arrival time for the fourth reflected peak. 


\subsection{Maximum overpressure}

To fit the maximum overpressure, two parameters were used as defined above: the geometrical parameter $\mathrm{H} / \mathrm{L}$ and the angle $\theta$.

Fig. 9 and Fig. 10, respectively, show the evolution of maximum overpressure for horizontal and vertical openings.

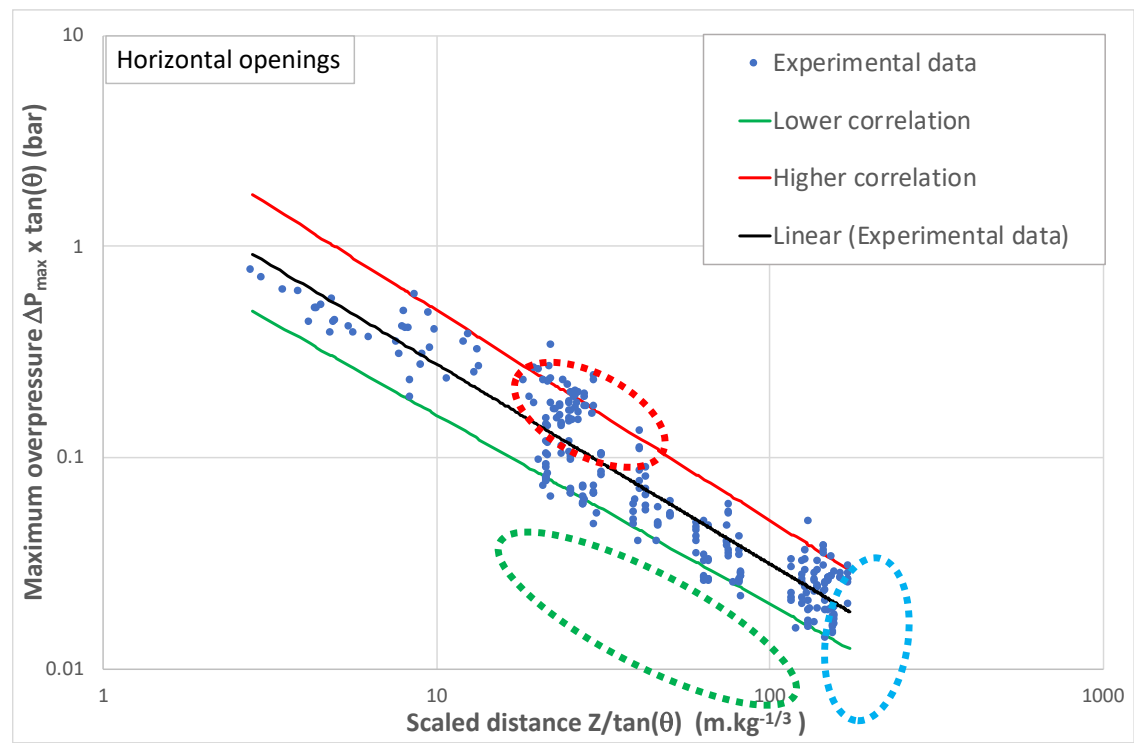

Figure 9: Correlation on the maximum overpressure for horizontal openings.

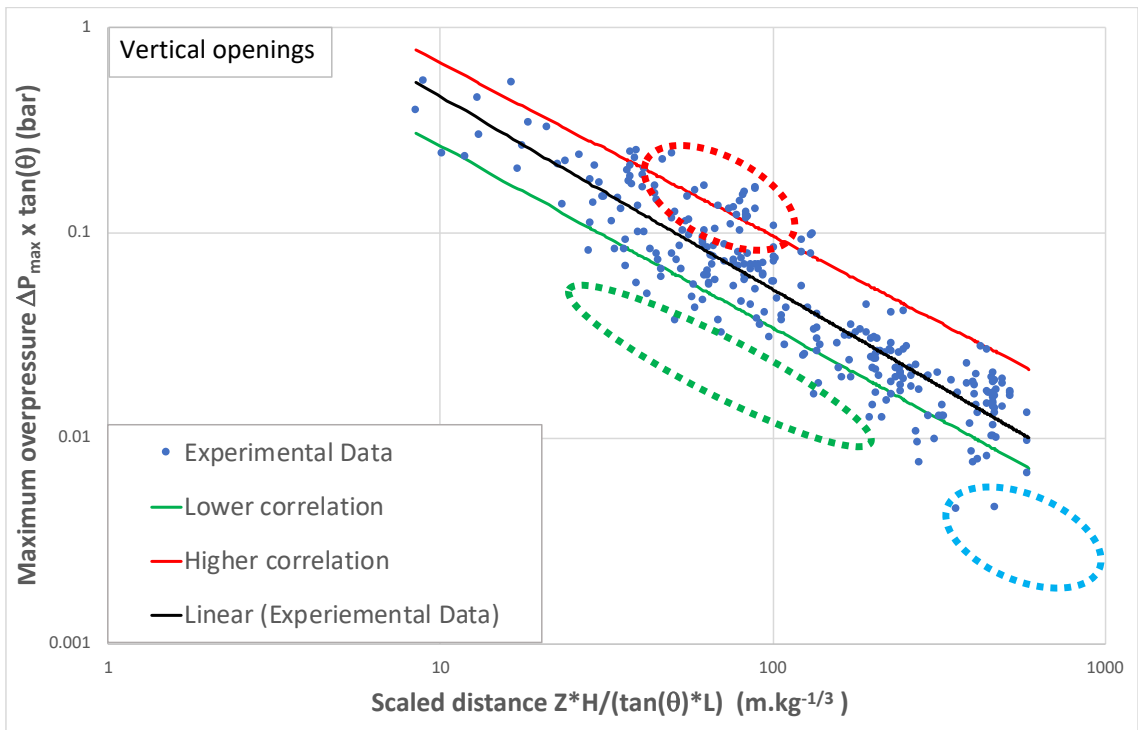

Figure 10: Correlation on the maximum overpressure for vertical openings. 
Table 3 reports the correlation laws for the maximum overpressure, as follows:

\[ \Delta \mathrm{P}_{\max } * \tan (\theta)=\mathrm{A}^{*}(\mathrm{X})^{-\mathrm{B}} \]
where for horizontal openings $\mathrm{X}$ represents $\frac{Z}{\tan (\theta)}$ and $\mathrm{X}=Z \times \frac{H}{L * \tan (\theta)}$ for vertical ones.

Table 3: Characteristics of correlations for maximum overpressure.

\begin{tabular}{|c|c|c|c|c|c|}
\hline & $\begin{array}{l}\text { Kind of } \\
\text { correlation }\end{array}$ & A & B & $\begin{array}{l}\text { Framework } \\
\left(m \cdot k g^{-1 / 3}\right)\end{array}$ & $\begin{array}{l}\text { Coefficient of } \\
\text { determination }\end{array}$ \\
\hline \multirow{3}{*}{$\mathbb{I}$} & Average & 2.443 & 0.946 & $\mathrm{X} \in(2.79 ; 173.26)$ & \multirow{3}{*}{$\mathrm{R}^{2}=0.875$} \\
\hline & Lower & 1.132 & 0.826 & $\mathrm{X} \in(2.79 ; 173.26)$ & \\
\hline & Higher & 12.578 & 1.325 & $\mathrm{X} \in(7.58 ; 173.26)$ & \\
\hline \multirow{3}{*}{2} & Average & 3.933 & 0.936 & $\mathrm{X} \in(8.43 ; 589.39)$ & \multirow{3}{*}{$\mathrm{R}^{2}=0.823$} \\
\hline & Lower & 1.035 & 0.733 & $\mathrm{X} \in(8.43 ; 589.39)$ & \\
\hline & Higher & 17.032 & 1.175 & $\mathrm{X} \in(16.44 ; 589.39)$ & \\
\hline
\end{tabular}

An analysis of the dispersion for overpressure laws was carried out. For the horizontal openings, the average deviation between experimental data and the correlation is $13 \%$. The maximum deviation is $51 \%$, obtained for sensor D6 with the charge in position P1 and PH-1344 as opening. This overpressure occurs very late in the pressure time history, at around $7.5 \mathrm{~ms}$, whereas the fourth overpressure peak for this sensor is obtained at $3.34 \mathrm{~ms}$. In general, for horizontal openings the average time for the maximum overpressure is $6.7 \mathrm{~ms}$; however, for the fourth arrival time the average is $3.7 \mathrm{~ms}$, with a maximum of $7.3 \mathrm{~ms}$. For the time of maximum overpressure, the greatest value is $24 \mathrm{~ms}$, i.e. extremely late in the history. This means that the maximum overpressure does not correspond to the first four overpressure peaks, but arrives later, due to reflection and confinement effects.

Three zones with a high degree of dispersion were identified, as shown in Fig. 9. The first one, in red, above the higher correlation, can be associated to the upper line (sensors $\mathrm{C} 1$ and $\mathrm{B} 7$ for P2 and sensors B4 and B2 for P1) only for small openings (PH-507 and PH-588). For the second one, in blue, the sensors affected are the low line of the back side (sensors B3-B5-D3-D6-C8). Nevertheless, for the part above the higher correlation it corresponds to large openings (larger than PH-1050), and small openings (lower than PH-588) for the part underneath the lower correlation. The third dispersion zone contains the nearest sensors, i.e. A8-C3-D1-D4-C6, only for small openings.

Finally, 27 points do not fall between the higher and lower correlations. Among these, only 5 are in direct view of the charge, so generally the maximum overpressure $(\Delta \mathrm{Pmax})$ of these transducers does not correspond to the first overpressure peak $\left(\Delta \mathrm{P}_{1}\right)$. In fact, for these points the average arrival time of the maximum overpressure is greater than for the others, $\operatorname{ta}_{\mathrm{Pmax}}=11.2 \mathrm{~ms}$.

For vertical openings, there are more points outside the lower and higher correlations. 40 points were analysed, and only six of them are in direct view of the charge. Moreover, 21 points are located underneath the lower correlation, and all are in indirect view. The results show that the error on maximum overpressure is smaller for sensors which are in direct view of the charge. Furthermore, only a few points outside the correlations are from the openings PV-1050 and PV-1344; dispersion is greater for small and medium-sized openings (from 
PV-300 to PV-840). This is due to the fact that diffraction is higher for small openings. The average deviation between experimental data and the correlation is $12 \%$. The maximum deviation is $55 \%$, obtained for sensor A8 with the charge in position P1 and opening PH- 840 . The time associated to this point is $11.4 \mathrm{~ms}$. In comparison with horizontal openings, the average arrival time of the maximum overpressure for vertical openings is greater, $\mathrm{t}=9.6 \mathrm{~ms}$, and the average arrival time for the fourth overpressure peak is $5.7 \mathrm{~ms}$. This shows that the maximum overpressure peak does not correspond to the beginning of the pressure time history, due once again to the confined geometry.

Three dispersion zones are located in Fig. 10. The red dispersion zone above the higher correlation corresponds to the back of the model (sensors B3-B4-B6-B7) for small to medium openings. The green and blue zones correspond to the sensors closest to medium openings, PV-588 to PV-1050. The green one represents sensors C4, C3, D4 and A7, and the blue one, sensor A8. These sensors are the most impacted by diffraction on the opening, regardless of the position of the charge.

\section{CONCLUSION}

The experimental campaign inside the model has enabled a large amount of data to be collected. It was the first step towards developing a new numerical code based on experimental laws. Inside the model, composed of two chambers, the emitting and the receiver chambers, reflections made the study complicated. The analysis of the pressure profile for different sensors or openings or even different positions of the charge help to elucidate the complex phenomena such as reflection and diffraction inside the model. The impact of the direction and the size of the opening were studied. Horizontal openings influence the overpressure for sensors on the top half of the receiver chamber. If the size increases, the first overpressure peak will be stronger, but the maximum overpressure will be lower. For vertical openings, some sensors are protected, as they are located at the entrance of the receiver chamber. For these sensors, the first overpressure peak is small. If the size of the opening increases the overpressure will increase, and the arrival time will decrease due to a less diffracted path of the shock wave. A significant difference of almost $3 \mathrm{~ms}$ on average was found in the arrival time of maximum overpressure between horizontal and vertical openings, due to diffraction. In general, the maximum overpressure in the receiver chamber for a charge position and an opening is obtained in the top back corner of the chamber.

Another point is that it was decided to focus on the first four overpressure peaks at the beginning. Some criteria were found to ensure an automatic and accurate selection of overpressure peaks. With these criteria, experimental correlations were found for the first four arrival times and the maximum overpressure. Three parameters were introduced to improve the quality of the laws: one represents the opening, the second is the angle of incidence, and the third is an angle that stands for the exposure of the opening to the charge. For each law, lower and higher correlations have been defined. A new experimental campaign will be carried out to find out laws for the first four overpressures. Two new positions of the charge will therefore be studied to ensure that the laws are correct as well as to improve them. The laws will then be introduced in a predictive code currently under development. The code has to be simple and to give the different parameters of the shock wave quickly. It must be able to calculate the path of the shock wave in a simple model composed of two chambers. Once finished, the code will be validated with experimental results.

ACKNOWLEDGEMENT

This work was funded by DGA Techniques Navales under contract $\mathrm{N}^{\circ} 15-86-0027$. 


\section{REFERENCES}

[1] Togashi, F., Baum, J.D., Mestreau, E., Löhner, R. \& Sunshine, D., Numerical simulation of long-duration blast wave evolution in confined facilities. Shock Waves, 20(5), pp. 409-424, 2010.

[2] van Erkel, A., Numerical Study and Extracted Model for Blast Jetting in Corridors, MABS21: Jerusalem, 2010.

[3] Salvado, F., Tavares, A., Teixeira-Dias, F. \& Cardoso, J., Confined explosions: The effect of compartment geometry. Journal of Loss Prevention in the Process Industries, 48, pp. 126-144, 2017.

[4] Ben-Dor, G., Shock Wave Reflection Phenomena, Springer: Berlin, 2007.

[5] Sugiyama, Y., Homae, T., Wakabayashi, K., Matsumura, T. \& Nakayama, Y., Numerical simulations of blast wave characteristics with a two-dimensional axisymmetric room model. Shock Waves, 27(4), p. 615-622, 2017.

DOI: 10.1007/s00193-016-0706-4.

[6] Mirova, O., Golovastov, S.V., Kotelnikov, A. Golub, V. \& Bazhenova, T., Multiple reflected shock wave in closed volume with granular screen. 31st International Symposium on Shock Waves, 2nd ed., Springer International Publishing: Cham, pp. 641-647, 2019.

[7] Teland, J.A., Hauge, K.O., Christensen, S.O. \& Børve, S., Numerical Simulation of Confined Blast, MABS 25: The Hague, 2018.

[8] Gebbeken, N., Larcher, M., Casadei, F., Solomos, G. \& Esteban, B., Safety of Metros Against Terrorist Attacks - Performance of Different Train Designs Subjected to Explosions, MABS 25: The Hague, 2018.

[9] Miura, A. et al., Reflection and diffraction phenomena of blast wave propagation in nuclear fuel cycle facility. JSME, 47(2), pp. 287-292, 2004.

[10] Gault, K., Etude de la propagation des ondes de choc en milieu confiné : Approche expérimentale, $\mathrm{PhD}$ thesis, Université d'Orléans, France, 2018. 